\title{
Diseño y construcción de una mano robótica para la enseñanza del alfabeto dactilológico universal para personas sordomudas
}

\author{
Daniel Alejandro Zúñiga Tenesaca*, Diego Miguel Andrade Zeas* \\ email: dzunigat@hotmail.com
}

\section{Resumen}

Este prototipo tiene como principal objetivo ayudar al aprendizaje de personas que tienen deficiencia auditiva, descrito de una mejor manera personas que siempre carecieron del sentido auditivo, mediante el diseño y la construcción de una mano robótica, capaz de realizar las señas del alfabeto dactilológico y que interactué con la persona mediante el reconocimiento de señas capturadas por una cámara web, para su posterior procesamiento por visión artificial en la plataforma de desarrollo National Instruments Labview 2009 todo esto en tiempo real.

La mano robótica será formada por diecisiete servomotores, para lograr los movimientos y los grados capaces de realizar una seña. Sus aplicaciones son innumerables ya que el prototipo esta desarrollado modularmente, cada parte puede ser aplicada en otros sistemas como el reconocimiento de imágenes, el control de servomecanismos, sistemas mecánicos. El prototipo como tal puede ser aplicado en el desarrollo de prótesis, manipulación de objetos mediante guantes sensoriales, estudios de señales biomecánicas para el control de la mano, etc.

Palabras clave: LabVIEW, servomotores, grilon, controlador, visión artificial, $18 \mathrm{~F} 4550$

\section{Abstract}

The prototype's main objective is to help people with learning of hearing loss, by designing and building a robotic band capable of performing the signs of finger spelling and interact with a person by recognizing signals captured by a webcam which will be processed in real time by means of the use of Labview 2009 - National Instruments vision development plataform.

The robotic hand is composed by 17 actuators to achieve the grades movements and able to make a sign. Its applications are countless since the prototype is developed modularly so each partcan be used in other systems such as image recognition, servo control, mechanical systems and the prototype itself can be used in: prostheses development of prostheses, objeect manipulation through sensorial gloves handling, the study of the biometrical signals of the band and so on.

Keywords: LabVIEW, servo, grilon. controller, artificial vision, $18 F 4550$

\footnotetext{
*Estudiantes de la Carrera de Ingeniería Electrónica - UPS - sede Cuenca Recibido: 30-julio-2011; Aprobado: 5-octubre-2011

Forma sugerida para citar: Zúñiga Tenesaca, Daniel. (2011). "Diseño y construcción de una mano robótica para la enseñanza del alfabeto dactilológico universal para personas sordomudas". INGENIUS. No 6, (julio/diciembre). pp. 67-84 .ISSN: 1390-650X
} 


\section{Introducción}

La mano robótica surgió de la necesidad de brindar una metodología de enseñanza del alfabeto dactilológico entretenida, diferente y que interactué con personas que tengan deficiencias auditivas, para que de esta manera se disminuya el tiempo de aprendizaje del alfabeto. Solo en la provincia del Azuay hay 1937 personas con discapacidades auditivas, habiendo en total en el Ecuador 35274 personas con esta deficiencia [1].

Existen muchos medios para el aprendizaje de las personas con esta discapacidad; una de las primeras etapas es el conocimiento del lenguaje de las señas, llamado también alfabeto dactilológico.

Este prototipo está diseñado de tal manera que posee diecisiete movimientos individuales $\mathrm{y}$ alrededor de cuarenta y un movimientos en forma conjunta, lo que nos da una variedad de aplicaciones como prótesis, control mediante guantes sensorizados o mediante visión artificial [2].

El complejo sistema se basa en doce servomotores que manejan las falanges de cada uno de los dedos, tres servomotores que sirven para movimientos de abducción y aducción y uno más para el movimiento rotacional del dedo pulgar.

Todo esto ha desencadenado una serie de estudios de esta parte del cuerpo humano para determinar las características esenciales y sus grados de movilidad [3].

Debido a la cantidad de huesos y falanges que contienen cada una de las articulaciones es necesario un análisis previo para establecer las pautas que posteriormente ayudaran a la construcción del prototipo [4].

La palma y cada una de las piezas de la mano están hechas de un material llamado grilon, por sus características resistentes a la temperatura y su menor desgaste por fricción gracias a su constitución [5]; estas piezas están unidas por hilos a los motores que hacen las veces de tendones para dar la movilidad suficiente para realizar las señas, que conjuntamente con un controlador de veinticuatro servos, realizado con un microcontrolador usb 18F4550, dan las secuencias de cada letra.

Por otra parte se dispondrá de un programa para que sirva de interfaz entre la mano robótica y el ser humano. Está realizado en la herramienta LabView; la persona tiene todas las letras del alfabeto, escoge una de ellas y la mano robótica la realiza, posteriormente el programa le pide a la persona que haga la misma seña. Mediante visión artificial con una cámara web se adquieren imágenes para el proceso de correlación entre las imágenes reales en tiempo real con imágenes patrones previamente guardadas en el programa.

\section{Materiales y métodos}

\subsection{Estructura y análisis de la mano humana}

La mano humana realiza funciones muy importantes como la función mecánica, sensitiva o hasta de comunicación. Es el principal órgano para la manipulación física del entorno [6]. La punta de los dedos contiene algunas de las zonas con más terminaciones nerviosas del cuerpo humano, son la principal fuente de in- 
formación táctil, es por eso que el sentido del tacto se asocia inmediatamente con las manos.

Se subdivide en tres partes: en la palma central (metacarpo) de la que surgen cinco dedos o falanges y la parte que la une con el antebrazo llamada muñeca (carpo) [6]. De esta manera la mano cuenta con veintisiete huesos incluyendo los de la muñeca, catorce falanges en los dedos, cinco metacarpianos y ocho carpianos, estos constituyen los grupos principales de articulaciones de la mano.

Las falanges son los huesos largos de los dedos, que presentan un cuerpo y dos extremos: la base y la cabeza.

El dedo pulgar tiene dos falanges: proximal y distal. El resto de los dedos tienen tres: las falanges proximal, media y distal (figura 1).

La base de la falange proximal se articula con la cabeza del hueso metacarpiano correspondiente [6]. La cabeza de la falange distal no se articula y se aplana formando una tuberosidad distal con forma de media luna, que se ubica bajo la almohadilla palmar del extremo del dedo.

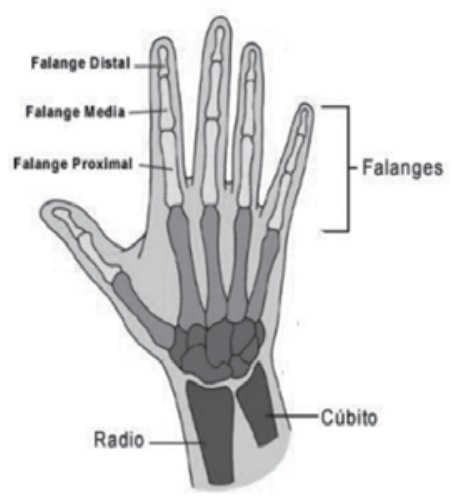

Fig. 1. Falanges de los dedos Fuente: dr. Carlos Azañero Inope
Los arcos de movilidad de las articulaciones de la mano determinarán las pautas para ver hasta cuántos grados una articulación puede realizar un movimiento, sin sufrir alguna lesión cuando se habla de un ser humano. De la misma manera servirá para que el prototipo tenga un límite de movimiento para que su parte mecánica no sea afectada, o también para que no realice movimientos innecesarios.

En el movimiento de extensión y flexión de la muñeca la amplitud media del movimiento es de $70^{\circ}$ para la extensión y de $80^{\circ}$ para la flexión (figura 2) [3].

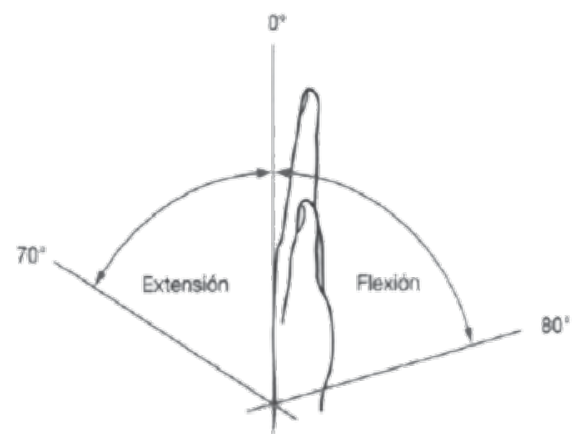

Fig. 2. Medición de la amplitud del movimiento de la muñeca

Fuente: René Cailliet, Anatomía funcional biomecánica.

En cambio la amplitud en las articulaciones metacapofalángicas es de $30^{\circ}$ a $45^{\circ}$ para la extensión y de $90^{\circ}$ para la flexión (figura 3) [3].

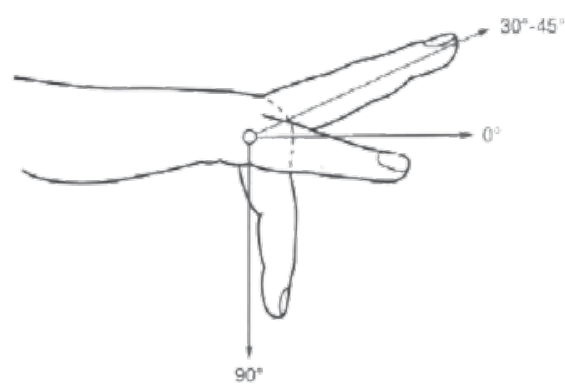

Fig. 3. Medición de la amplitud del movimiento de la articulación metacarpofalángica Fuente: René Cailliet, Anatomía funcional biomecánica. 
La flexión y extensión de las falanges distal y media (figura 4), se lo realiza juntando los dedos en movimiento continuo y tocan la palma aproximadamente hasta el nivel del surco palmar distal. [3]

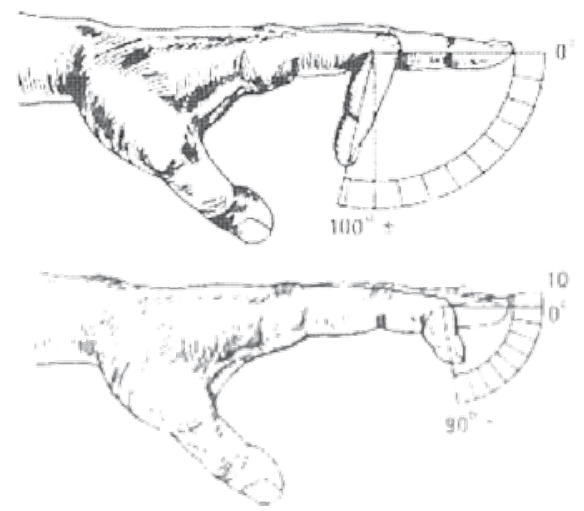

Fig. 4. Flexión y extensión de las falanges distal y media Fuente: http://redalyc.uaemex.mx/pdf/614/61411404.pdf

La abducción y aducción se miden a partir de la línea axial de la mano, todos los dedos se separan en arcos de aproximadamente $20^{\circ}$ [3], mientras que en aducción se juntan y tocan entre sí (véase figura 5).

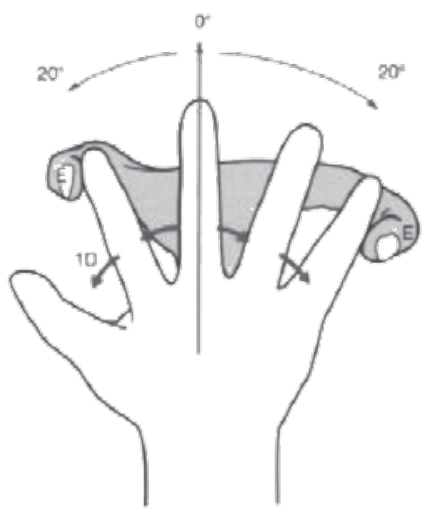

Fig. 5. Aducción y abducción de los dedos Fuente: René Cailliet, Anatomía funcional biomecánica
El pulgar puede abducirse en el plano de la palma $\left(0^{\circ}\right)$ o en ángulo recto en la abducción palmar (AP) hasta los $70^{\circ}$ (figura 6) [3].

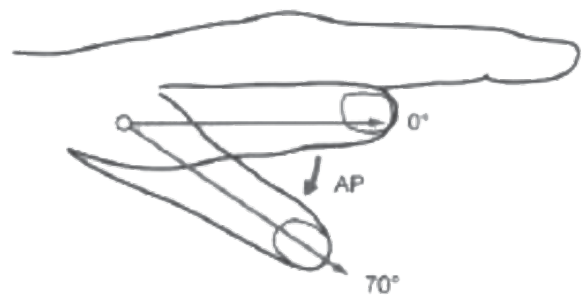

Fig. 6. Abducción del pulgar

Fuente: René Cailliet, Anatomía funcional biomecánica.

En el pulgar, la extensión consiste en los movimientos de alejamiento del lado radial del índice en la palma de la mano. La abducción es el movimiento de alejamiento de la palma en un plano perpendicular al plano de dicha palma. La flexión es el movimiento de alejamiento de la palma hacia el lado cubital [6]. Los dedos 2, 3 y 4 se flexionan hacia el adelante. En el quinto dedo la extensión involucra a todas las falanges. La abducción se realiza mediante el alejamiento de la palma a lo largo de su propio plano. La flexión es de $90^{\circ}$ en la articulación metacarpofalángica (figura 7).

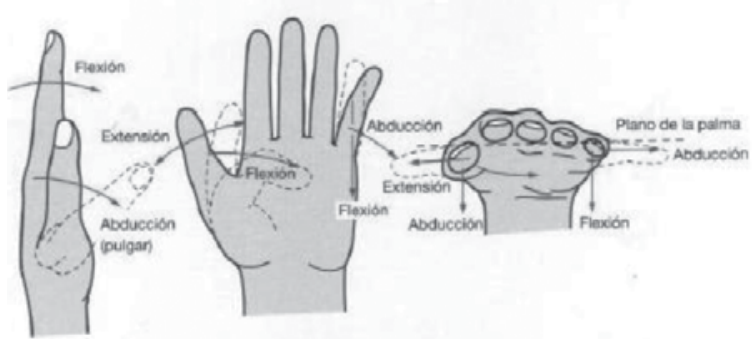

Fig. 7. Movimientos del pulgar e índice Fuente: René Cailliet, Anatomía funcional biomecánica

El rango de movilidad de las articulación en la flexión y extensión decrecen cuando la abducción y aducción se incrementan y viceversa 
desde la posición central del dedo, esto debido a la estructura de los metacarpianos.

El pulgar también tiene el movimiento de circunducción, este movimiento consiste en que el dedo describe un cono cuyo vértice está en la articulación y su base en la extremidad distal de esa parte y no necesita rotación [8].

Otra parte muy importante son los grados de libertad, los cuales nos dan una idea de la relación de fuerza/peso.

Cada dedo cuenta con tres falanges, falange distal, media y proximal, mientras que el dedo pulgar solo cuenta con la falange distal y proximal.

La cantidad de grados de libertad (GDL) permiten múltiples configuraciones de aprehensión y manipulación que van incrementando en potencialidad al cambiar los planos de trabajo, ya que poseen articulaciones tipo bisagra que permiten dicha característica. [7]

Tabla 1. Estudio antropométrico de la mano por Garrett

\begin{tabular}{|c|c|c|c|}
\hline Falange & Proximal & Media & Distal \\
\hline Pulgar & 17.1 & - & 12.1 \\
\hline Índice & 21.8 & 14.1 & 8.6 \\
\hline Medio & 24.5 & 15.8 & 9.8 \\
\hline Anular & 22.2 & 15.3 & 9.7 \\
\hline Meñique & 17.2 & 10.8 & 8.6 \\
\hline
\end{tabular}

Para el análisis estático de los dedos se debe tener en cuenta las longitudes de las falanges, el peso, la gravedad, el punto de inserción del músculo, el centro de articulación y puntos de contacto entre las falanges.

Se han realizado trabajos relacionados con el estudio antropométrico de la mano y las falanges, en la siguiente tabla se muestra el estudio efectuado por Garrett [7] donde se detalla la relación en longitud que existe entre los dedos con respecto al porcentaje de la longitud total de la mano.

En la cinemática de la mano, es necesario manejar treinta variables mecánicas: seis por cada dedo, tres componentes de fuerza y tres componentes de momento. Es posible manipular la fuerza aplicada en cada uno de los dedos, así como cambiar la cinemática de agarre, usando diferentes posiciones en las falanges de los dedos.

\subsection{Diseño de la mano robótica}

Los parámetros a considerar para un diseño lo más semejante a la anatomía humana se debe considerar la forma y el número de falanges necesarias para asemejarse lo mayor posible a una mano humana.
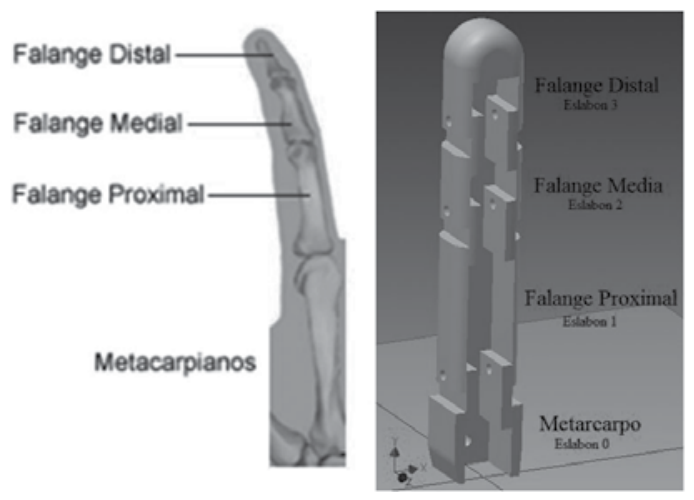

Fig. 8. Dedo índice Fuente: Autoría propia

En las figuras 8 y 9 se pueden notar las falanges diseñadas de tal forma que estas se asemejen la anatomía humana, por lo que se muestra el dedo índice y el pulgar, ya que basta con modificar las dimensiones de cada eslabón para tener los demás dedos es decir el dedo anular, medio y meñique. 
Se debe considerar que la cantidad de movimientos es de suma importancia para la determinación de actuadores.
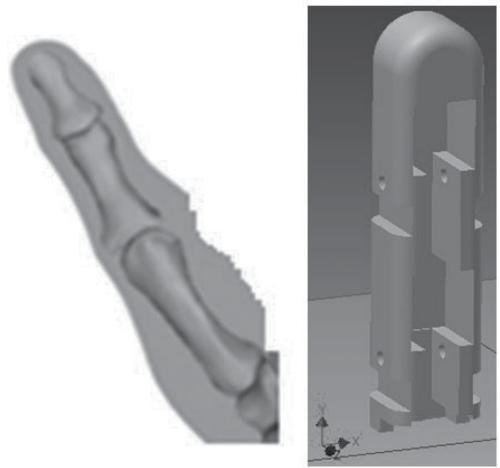

Fig. 9. Dedo pulgar Fuente: Autoría propia

\section{Diseño de articulaciones}

Para el diseño de las articulaciones, en primer lugar debemos notar las articulaciones que posee la mano, en la figura 10 se observa que la mano humana tiene ocho tipos de articulaciones, teniendo en consideración hasta la muñeca.

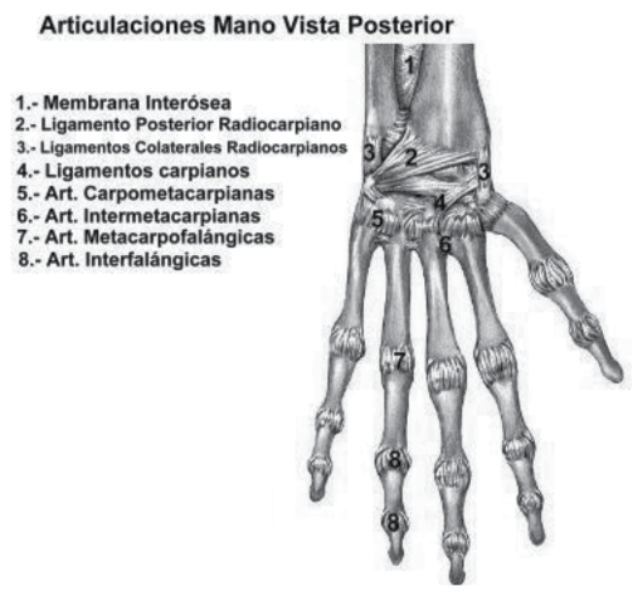

Fig. 10. Articulaciones de la mano Fuente: http://www.anatomiahumana.ucv.cl/efi/modulo4.html
Como se requiere un movimiento rotacional en un solo eje, para las falanges se opta por colocar un eje cilíndrico entre cada falange, el mismo que es de bronce ya que este material tiene mayor resistencia al rozamiento. La articulación se la muestra en la figura 11.

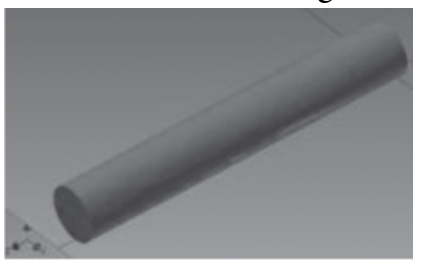

Fig. 11. Eje de bronce Fuente: Autoría propia

La palma se la plantea de tal forma que posea los espacios para cada uno de los dedos, además del número necesario de agujeros para los ductos, que guiarán cada uno de los hilos para lograr el movimiento (figura 12)

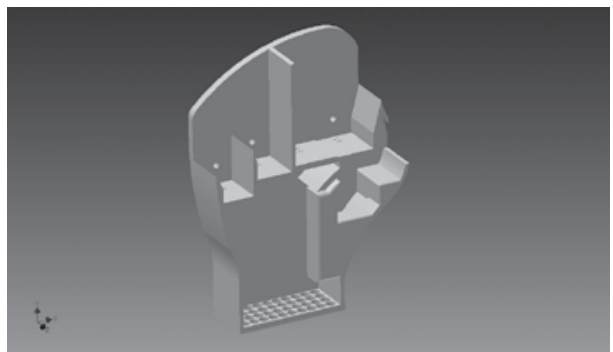

Fig. 12. Palma

Fuente: Autoría propia

Una vez diseñada cada una de las partes se las procede a ensamblar en $\mathrm{CAD}$, por lo que podemos notar un diseño compacto, de tal forma que la inercia que se genera no afecte al conjunto en general.

En la figura 13 se aprecia el diseño propuesto completo, el mismo que se construirá con materiales existentes en nuestro medio. 


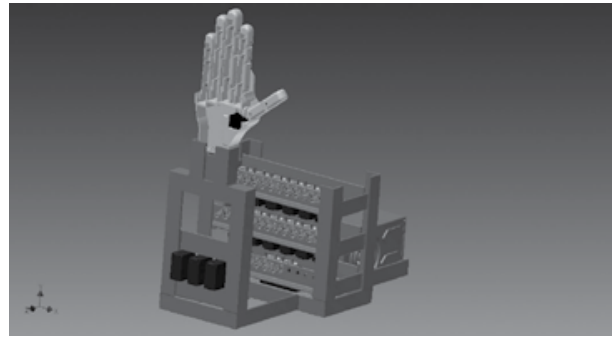

Fig. 13. Ensamble total

Fuente: Autoría propia

\subsection{Geometría de la mano robótica didáctica}

El dedo a analizar plantea el siguiente plano en donde podemos observar tanto las características físicas, como la disposición de los ejes de análisis. Este esquema puede adaptarse a cualquiera de los dedos modificando sus variables articulares y sus dimensiones.

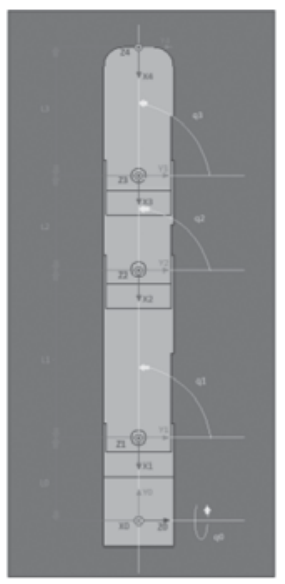

Fig. 14. Plano del dedo genérico Fuente: Autoría propia

Entonces tendremos el eje Y0 situado sobre el eje paralelo al dedo; el eje Z0 situado sobre el eje de rotación y el eje X0 situado de manera que forme un sistema dextrógiro. Para los demás sistemas coordenados, debido a que cambia el eje de rotación, cambia también su disposición en el plano de análisis teniendo en este caso $\mathrm{X}$ en sentido negativo paralelo al dedo en vista lateral, $Z$ situado sobre el eje de rotación en dirección saliente e $Y$ situado de modo que también forme un sistema dextrógiro con los dos anteriores.

Ahora, las dimensiones del dedo en análisis son las siguientes:

\begin{tabular}{|l|}
\hline L0 $=17 \mathrm{~mm}$ \\
\hline L1 $=34 \mathrm{~mm}$ \\
\hline L2 $=19 \mathrm{~mm}$ \\
\hline L3 $=26 \mathrm{~mm}$ \\
\hline L0 $=17 \mathrm{~mm}$ \\
\hline
\end{tabular}

Las variables articulares inmiscuidas en el modelo son solamente angulares (movimiento rotacional) y se encuentran definidas por: $q^{0}$
$q_{1}$
$q^{2}$
$q^{3}$

Entonces, una vez definida la geometría inmiscuida del dedo en cuestión, pasamos al análisis cinemático del mismo.

\subsubsection{Cinemática directa}

En este punto definiremos el análisis de la cinemática o movimiento del robot respecto a un sistema de referencia.

Este análisis corresponde a un tipo específico de cinemática mediante el cual se puede determinar la posición y orientación de un robot, respecto a un sistema de referencia, conociendo únicamente los valores de las variables articulares que actúan sobre dicho robot [11]. En nuestro caso estas variables articulares serán simplemente ángulos.

Existen varias formas de resolver dichos sistemas, pero por razones de facilidad y sobre 
todo precisión, haremos uso del método de matrices de transformación homogéneas.

Entonces, para ello será necesaria una revisión previa de dichas matrices. Por tanto decimos que una matriz de este tipo es de la forma [9]:

$$
T=\left[\begin{array}{cc}
R_{3 x 3} & P_{3 x 1} \\
f_{1 x 3} & w_{1 x 1}
\end{array}\right]
$$

De donde: $\mathrm{R}$ será la matriz de rotación, $\mathrm{P}$ será la matriz de traslación, f será la matriz de perspectiva, que para el caso de los robots es 0 por su propia mecánica y w se constituye como la matriz de escalado, la cual adopta el valor de 1 para el caso del robot [9].

Para la determinación de la cinemática se usará un método bastante práctico, conocido como el algoritmo de Denavit-Hartenberg, el mismo que establece reglas de desarrollo. Mediante este algoritmo se halla $\mathrm{T}$ por medio del producto de $\mathrm{A}_{\mathrm{i}-1}^{\mathrm{i}}$ las cuales son matrices relacionantes de eslabones contiguos; es decir, al multiplicar dichas matrices ${ }_{i-1} \mathrm{~A}^{\mathrm{i}}$ en orden ascendente se logra relacionar el sistema de coordenadas primitivo con el final, posibilitando de esta forma un control sobre cualquier robot al que se le aplique este algoritmo [9].

Ahora, la matriz A se determina de la siguiente forma:

${ }^{i-1} A^{i}$
$=\left[\begin{array}{ccc}\cos \theta_{i} & -\cos \alpha_{i} \cdot \sin \theta_{i} & \sin \alpha_{i} \cdot \sin \theta_{i} \\ \sin \theta_{i} & \cos \alpha_{i} \cdot \cos \theta_{i} & -\sin \alpha_{i} \cdot \cos \theta_{i} \\ 0 & \sin \alpha_{i} & \cos \alpha_{i} \\ 0 & 0 & 0\end{array}-\right.$

Cada una de las incógnitas aquí expuestas se denominan parámetros de Denavit-Hartenberg, que no son más que parámetros relativos al tamaño y forma del eslabón, estos se encuentran definidos como:
$A^{i}$ que es la distancia entre los ejes de dos articulaciones contiguas de un mismo eslabón medidas en línea recta.

$\alpha_{i}$ que es el ángulo al cual se encuentra girado uno de los ejes de un eslabón respecto a su eje inmediato predecesor del mismo eslabón.

$d_{i}$ es la distancia que existe entre dos caras símiles de ejes de articulaciones contiguas; es decir, entre los ejes final de un eslabón predecesor y el inicial del eslabón siguiente.

$\theta_{i}$ es el ángulo que está rotado un eslabón predecesor respecto de su contiguo, o mejor dicho, el ángulo que existe entre dos eslabones contiguos.

Una vez conocido esto, procedemos a la aplicación del algoritmo propiamente dicho. Esto resulta en la siguiente tabla de los parámetros de Denavit-Hartenberg, conocidos como parámetros D-H:

Tabla 2.Valores obtenidos para los parámetros D-H de nuestro prototipo en análisis

\begin{tabular}{|l|l|l|l|l|}
\hline Artic & $\boldsymbol{\theta}$ & $\mathbf{d}$ & $\mathbf{a}$ & \multicolumn{1}{c|}{$\boldsymbol{\alpha}$} \\
\hline 1 & $-(90-\mathrm{q} 0)$ & 0 & $-\mathrm{L} 0$ & 90 \\
\hline 2 & $-(90-\mathrm{q} 1)$ & 0 & $-\mathrm{L} 1$ & 0 \\
\hline 3 & $-(90-\mathrm{q} 2)$ & 0 & $-\mathrm{L} 2$ & 0 \\
\hline 4 & $-(90-\mathrm{q} 3)$ & 0 & $-\mathrm{L} 3$ & 0 \\
\hline
\end{tabular}

Cabe recalcar que se ha introducido una variable articular $\mathrm{q}_{0}$, esta será útil solamente en los casos donde se analicen dedos que posean dicho grado de libertad (movimiento hacia los lados), que es el caso del anular, índice y pulgar, en los demás casos, su consideración se anula. Entonces las matrices A para cada eslabón serán:

${ }_{0} A^{1}=\left[\begin{array}{cccc}\sin q_{0} & 0 & -\cos q_{0} & -L_{0} \sin q_{0} \\ -\cos q_{0} & 0 & -\sin q_{0} & L_{0} \cos q_{0} \\ 0 & 1 & 0 & 0 \\ 0 & 0 & 0 & 1\end{array}\right]$ 


$$
\begin{aligned}
{ }_{1} A^{2} & =\left[\begin{array}{cccc}
\sin q_{1} & \cos q_{1} & 0 & -L_{1} \sin q_{1} \\
-\cos q_{1} & \sin q_{1} & 0 & L_{1} \cos q_{1} \\
0 & 0 & 1 & 0 \\
0 & 0 & 0 & 1
\end{array}\right] \\
{ }_{2} A^{3} & =\left[\begin{array}{cccc}
\sin q_{2} & \cos q_{2} & 0 & -L_{2} \sin q_{2} \\
-\cos q_{2} & \sin q_{2} & 0 & L_{2} \cos q_{2} \\
0 & 0 & 1 & 0 \\
0 & 0 & 0 & 1
\end{array}\right]
\end{aligned}
$$

Para comprobación de la validez de estas ecuaciones proporcionamos ángulos aleatorios y los comprobamos con los resultados obtenidos en Matlab. Entonces al aplicar q1 $=50^{\circ}$, $\mathrm{q} 2=30^{\circ}, \mathrm{q} 3=75^{\circ}$ obtenemos mediante nuestro algoritmo:

$$
\begin{aligned}
& X=0 \\
& Y=18,5901 \\
& Z=55,7449
\end{aligned}
$$

Ahora mediante nuestros bloques de Simulink al ingresar los siguientes ángulos:

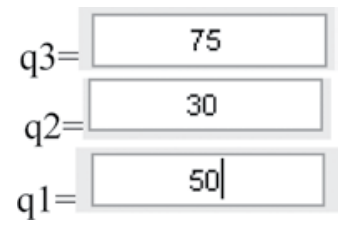

Se obtienen los siguientes resultados en SimMechanics:

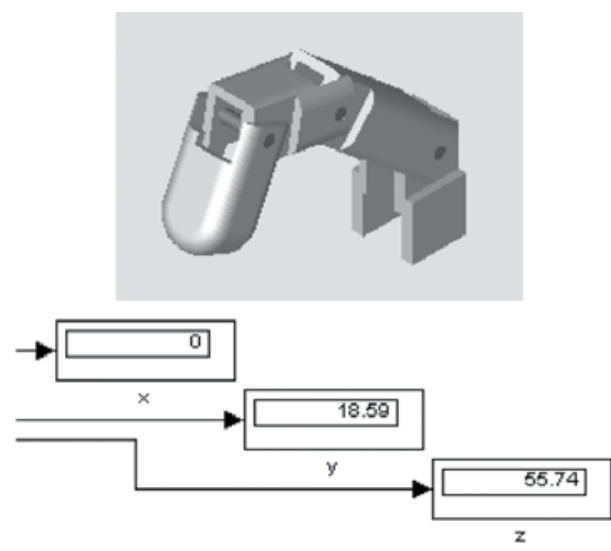

Fig. 15. Resultados obtenidos mediante la aplicación de la cinemática directa Fuente: Autoría propia
Entonces se evidencia la veracidad de nuestras ecuaciones dado que los resultados obtenidos son idénticos.

\subsubsection{Cinemática inversa}

Para el caso de la cinemática inversa se hará un análisis de tipo geométrico, debido a que la aplicabilidad del algoritmo D-H (DenavitHartenberg) usado en la sección anterior está restringido para tres grados de libertad, y en el caso de nuestro modelo de análisis o robot se usan hasta cuatro.

El análisis de tipo geométrico no es más que un tipo específico de análisis cerrado, en donde se hace necesario el conocimiento de la geometría del robot y se tiene como dato la posición que se quiere alcanzar. Este análisis se evidencia como necesario debido a los problemas que se presentan en esta clase de cinemática como es la existencia de múltiples soluciones, resolución no sistemática, existencia de ecuaciones no lineales, etc. [11]

Entonces, como se aprecia se hace necesaria una revisión de conceptos trigonométricos útiles que permitan relaciones entre los diversos segmentos de prototipo en cuestión.

La ecuación que nos resulta del análisis geométrico es la siguiente:

$\mathrm{S}=\operatorname{solve}\left(\mathrm{y}-\left(\mathrm{l}_{0}+\mathrm{l}_{1}^{*} \sin \left(\mathrm{q}_{1}\right)+\mathrm{l}_{2} * \sin \left(\mathrm{q}_{2}\right)+\mathrm{l}_{3}^{*} \sin \left(\mathrm{q}_{3}\right)\right)\right.$, $\mathrm{z}-\left(\mathrm{l}_{1}^{*}\right.$ ' $\left.\left.\cos \left(\mathrm{q}_{1}\right)+\mathrm{l}_{2}^{*} \cos \left(\mathrm{q}_{2}\right)+\mathrm{l}_{3}^{*} \cos \left(\mathrm{q}_{3}\right)\right), \mathrm{p}_{\mathrm{i}}-\mathrm{q}_{1}-\mathrm{q}_{2}\right)$

Esta ecuación nos permitirá hallar la cinemática inversa inmiscuida en nuestro prototipo, entonces al simular dicha ecuación para los siguientes valores:

$$
\begin{aligned}
& 1_{0}=17 \\
& 1_{1}=34 \\
& 1_{2}=19
\end{aligned}
$$




$$
\begin{gathered}
\mathrm{l}_{3}=26 \\
\mathrm{x}=0 \\
\mathrm{y}=96 \\
\mathrm{z}=0
\end{gathered}
$$

Obtenemos los siguientes resultados en cuanto a variables articulares se refiere

$\mathrm{q}_{0}=0$

$\mathrm{q}_{1}=90,000000000000003122521083018737$

$\mathrm{q}_{2}=90,000000000000003122492513291159$

$\mathrm{q}_{3}=90,000000000000003122498556887378$

Para la simulación de estos resultados, acudimos de nuevo a nuestros bloques de Simulink en los cuales ingresamos los ángulos propuestos:

$\mathrm{q}_{3}=\mathrm{q}_{2}=\mathrm{q}_{1}=90$

A través de SimMechanics se obtienen los siguientes resultados que confirman la validez de la ecuación.

Fig. 16. Resultados obtenidos mediante la aplicación de la cinemática inversa.
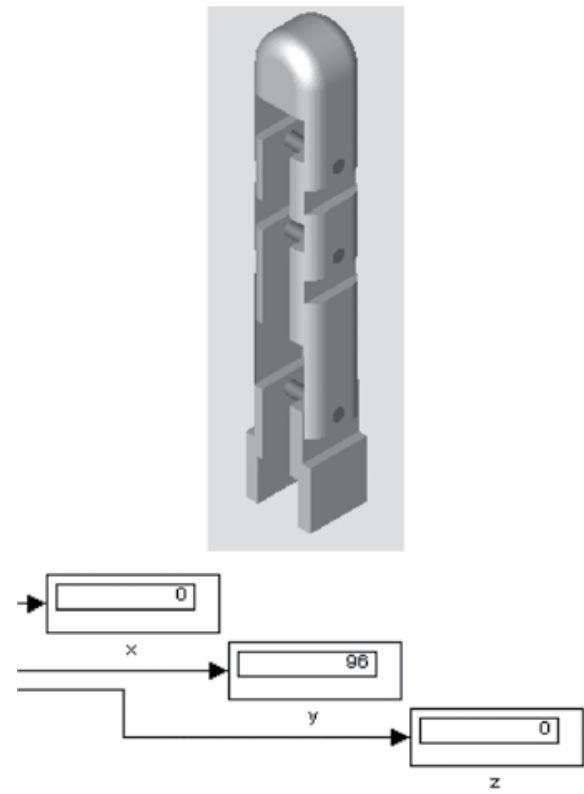

Fuente: Autoría propia

\subsection{Construcción del prototipo}

Para la construcción del prototipo se requieren materiales tales como hierro, suelda grilon, calibradores, forros, entre otros. En el diseño se han desarrollado cada uno de los planos, por lo que en esta sección describiremos una secuencia de imágenes ya que cada uno de los planos normalizados ocuparía un número considerable de hojas por la cantidad de piezas.

En la figura 17 se observa la palma conjuntamente con el controlador el mismo que se detallará posteriormente.

Fig. 17. Palma y controlador Fuente: Autoría propia

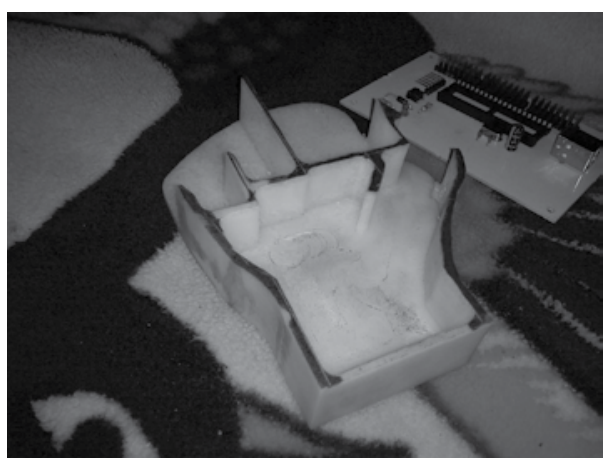

En la siguiente secuencia de fotografias (figura 18) se muestra los dedos armados en la palma y también un contraste con nuestra mano para constatar el movimiento de aducción y abducción. Como siguiente paso la palma se la va adecuar para dirigir cada uno de los hilos encargados de transmitir el movimiento desde los servomotores.

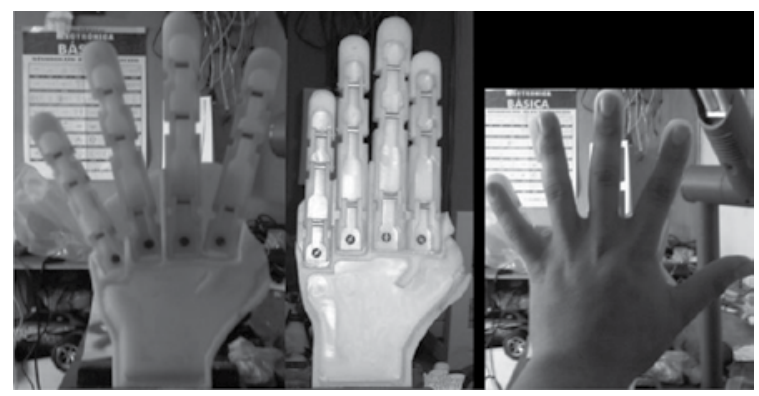

Fig. 18. Cuatro dedos montados en la palma Fuente: Autoría propia 
Para cada falange se construyen placas metálicas que sujetarán cada hilo nailon a cada una de la falange, ajustado por medio de tornillos (figura 19).

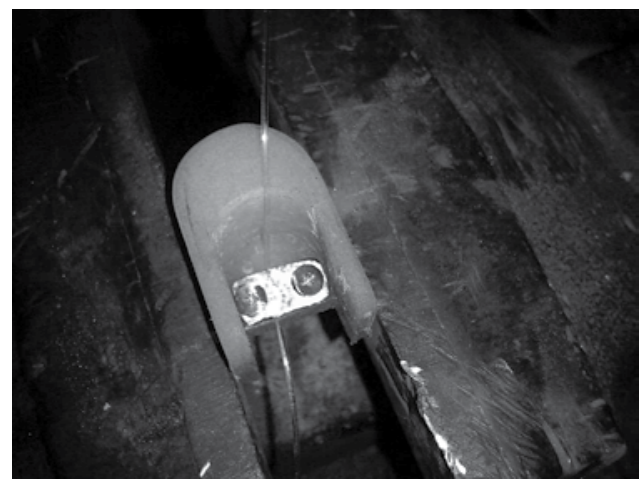

Fig. 19. Ajuste de hilo nailon Fuente: Autoría propia

Cada una de las piezas debe ser bien ensamblada de tal forma que no existan fallos en el funcionamiento de cada uno de los de los dedos; se debe considerar que el prototipo final está diseñado para realizar pruebas. En la figura 20 se puede mostrar ya el prototipo construido y ensamblado.

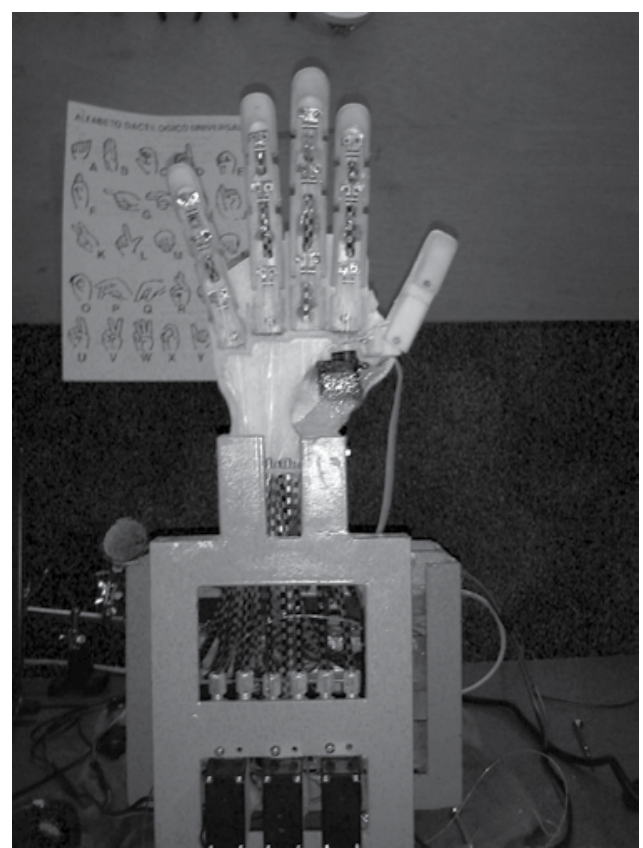

Fig. 20. Prototipo final Fuente: Autoría propia

\subsection{Controlador}

El controlador se implementará en un microcontrolador PIC $18 \mathrm{f} 4550$ (figura 21), denominado PIC USB, del cual vamos a utilizar sus interrupciones para desarrollar el controlador de veinticuatro canales.

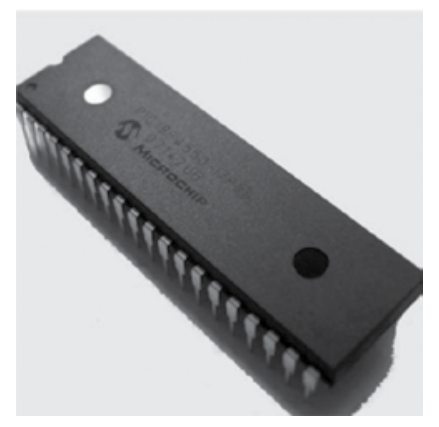

Fig. 21. Pic $18 \mathrm{f} 4550$

Fuente: http://www.alibaba.com/productgs/384176481/PIC18F4550.html

Como se sabe, para cambiar la posición del servomotor se debe modificar el ancho de pulso que debe variar con frecuencia igual a $50 \mathrm{~Hz}$, en la figura 22 se observa los anchos de pulsos para las diferentes posiciones.

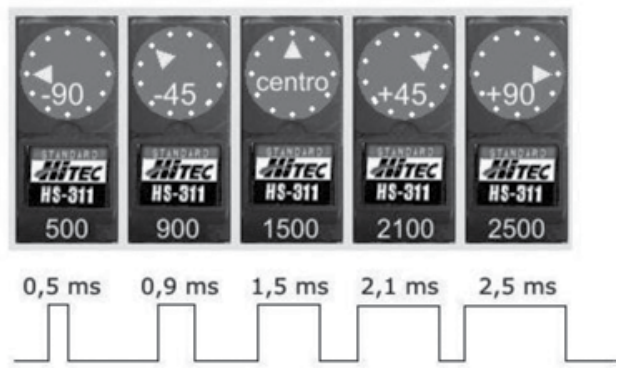

Fig. 22. Anchos de pulsos para controlar el servomotor Fuente: http://www.alibaba.com/productgs/384176481/PIC18F4550.html

Para desarrollar el algoritmo en primera instancia debemos calcular el periodo para cada servomotor:

$T=\frac{1}{F}=\frac{1}{50}=20 \mathrm{~ms}$

Esto quiere decir que cada $20 \mathrm{~ms}$ debe existir el tiempo en alto necesario para la posición 
que se requiera, como sabemos que el máximo pulso que se le puede dar a un servomotor es de 2,5 ms dividimos el periodo para esta ventana de tal forma que nos dará el número de servomotores que podemos manejar simultáneamente dentro de este periodo.

$$
\# \text { de Servos }=\frac{20 \mathrm{~ms}}{2,5 \mathrm{~ms}}=8 \text { servos }
$$

A cada tiempo de $2,5 \mathrm{~ms}$ se denomina «ventana», entonces hay que considerar que esta debe cumplir independientemente del ancho de pulso necesario para ubicar al servomotor (figura 23.)

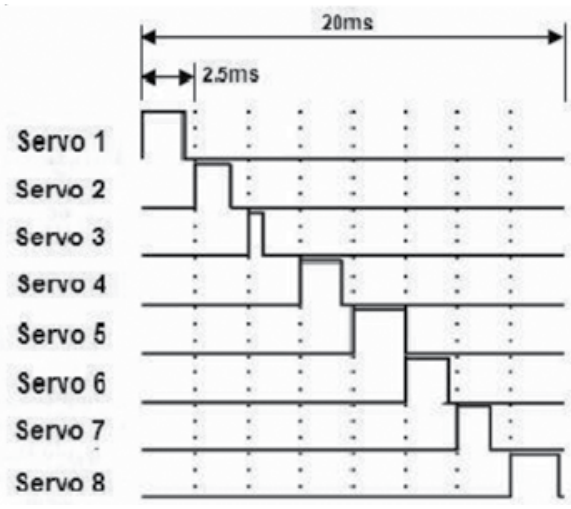

Fig. 23. Ventanas para controlar Fuente: http://cybertesis.upc.edu.pe/ upc/2009/torres_gj/html/TH.5.html

Hay que notar antes de activar el siguiente servomotor se debe cumplir la ventana de $2,5 \mathrm{~ms}$, por ejemplo si se posiciona el primer servomotor a 0 grados, es decir ancho de pulso de 0,5 ms los 2 restantes deben permanecer en bajo antes de activar siguiente servomotor (figura 23).

Para lograrlo se usarán las interrupciones por temporizador, para cada ocho servos vamos a utilizar uno, por lo que para los veinticuatro servomotores necesitamos tres temporizadores, el PIC utilizado posee cuatro temporizadores de los cuales tres de ellos son de 16 bits, lo que nos sirve para tener una exactitud buena.
El parámetro fundamental para realizar el cálculo para cada servomotor es el tick, es decir el tiempo que demora en incrementar en uno el temporizador, por lo cual expresaremos el número de ticks para el tiempo en alto para cada uno de los servos.

Por ejemplo si todos tuviesen que estar a 90 grados sus PWM deberían de ser de 1,5 ms en alto:

$$
T x=\frac{1.5 \mathrm{~ms}}{0.0833 \mathrm{us}}=18007 \text { Ticks }
$$

Entonces habilitamos la interrupción por desbordamiento de temporizador; la primera vez que desborde ponemos en alto el pin correspondiente al primer servo y precargamos el valor del temporizador 1 con 18007 ticks antes del desborde, que se produce a los 65535 ticks (216-1), con lo que ponemos el temporizador a 65535 - $18007=47$ 528, de esta manera obtendremos la interrupción cuando transcurran esos 18007 ticks.

Cuando la interrupción se activa, y entramos por segunda vez tenemos que prefijar el temporizador para que se cumpla la ventana de 2,5 ms para activar el siguiente servomotor. En nuestro ejemplo para ubicar el servomotor a 90 grados utilizamos $1,5 \mathrm{~ms}$ o 18007 ticks, por consiguiente tendremos que esperar 2,5 $\mathrm{ms}-1,5 \mathrm{~ms}=1 \mathrm{~ms}$ para activar el siguiente servomotor.

Entonces vamos a precargar de nuevo el tem-

$$
T x=\frac{1 m s}{0.0833 u s}=12004 \text { Ticks }
$$

porizador con $65535-12004=53530$ y la interrupción se activara nuevamente en $1 \mathrm{~ms}$.

A raíz de la segunda interrupción activamos el siguiente servo y procedemos similarmente que el anterior caso. 


\subsection{Programa de interfase}

Para la adquisición del video en tiempo real se facilita gracias al Vision Acquisition que posee la opción de escoger el tipo de cámara, la resolución y parámetros como contraste iluminación, etc. (véase figura 24).

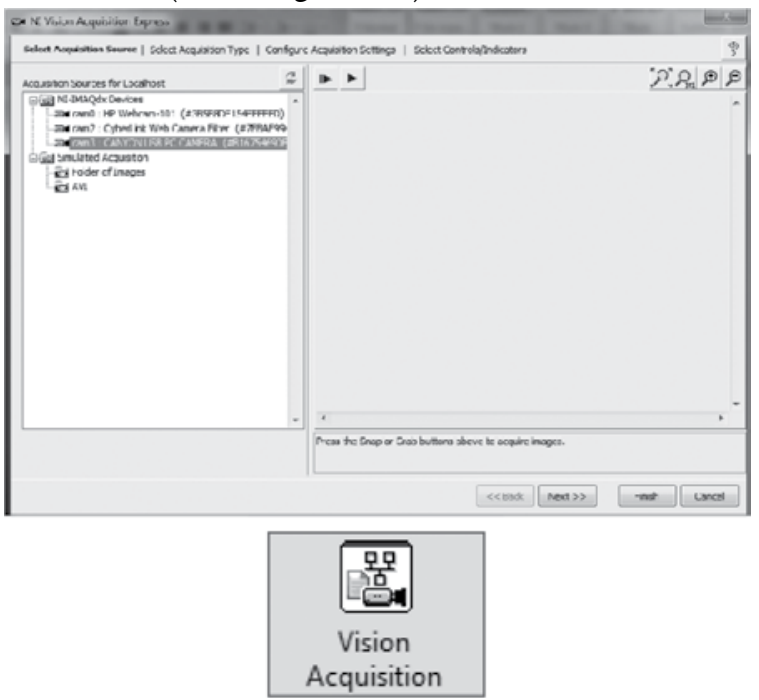

Fig. 24. Panel Vision Acquisition Fuente: Diego Andrade (autoría)

La estructura del programa se basa, en primer lugar, en imágenes patrones con una resolución de $320 * 240$ pixeles para que el proceso de reconocimiento no sea tan pesado. Se tiene tres imágenes patrón por cada letra, es decir un total de 78 imágenes para su reconocimiento (véase figura 25).

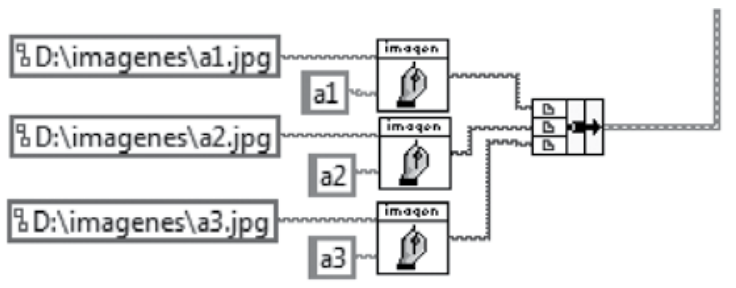

Figura 25 Ingreso de imágenes patrón Fuente: Diego Andrade (Autoría)

Dentro del bloque imagen se tiene un tratamiento a la letra patrón como es la escala de grises y sobre todo un VI de aprendizaje geométrico llamado Learn Geometric Pattern que es el encargado de aprender el contorno de imagen para un posterior procedimiento de búsqueda y correlación. La imagen resultante es guardada en una imagen de muestra para optimizar recursos de memoria (véase figura 26).

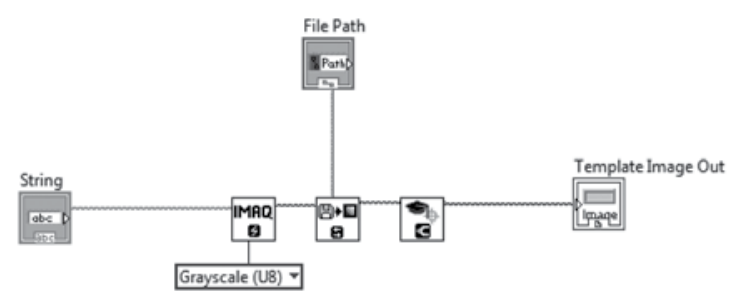

Fig. 26. Proceso imagen patrón Fuente: Diego Andrade (autoría)

Después las imágenes son cargadas a un bloque en donde ingresan los patrones con la imagen real y la imagen real pero en escala de grises, esto se hace para mantener la imagen real en RGB sin alteraciones. (Véase Figura 27)

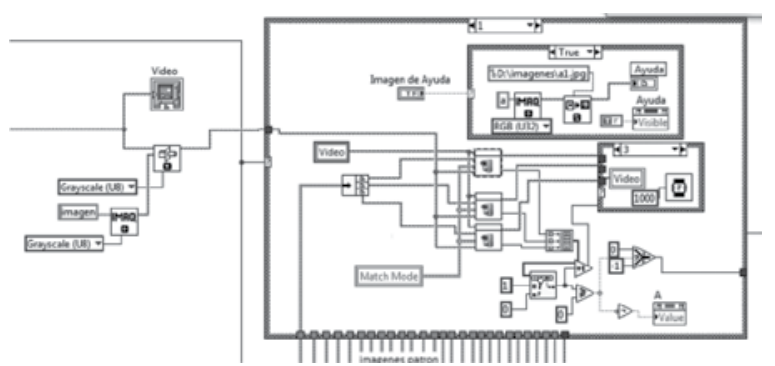

Fig. 27. Módulo para el reconocimiento de señas Fuente: Diego Andrade (autoría)

En este bloque lo que se realiza es mediante el match mode para controlar ámbitos como rotación, escala y oclusión para la posterior búsqueda del patrón en la imagen en tiempo real en escala de grises. La búsqueda del patrón es implementada mediante la herramienta Match Geometric Pattern que realiza la búsqueda de un patrón en una imagen de inspección que en nuestro caso es la imagen en tiempo real en escala de grises con el control del match mode, devolviéndonos un factor de correla- 
ción de dos valores 0 y 1 , siendo 1 si encontró similitudes entre las imágenes. Otros factores que ingresan es el número de objetos a reconocer que en nuestro caso es 1 y el mínimo valor resultante de la correlación, que por defecto es 800 (véase figura 28).

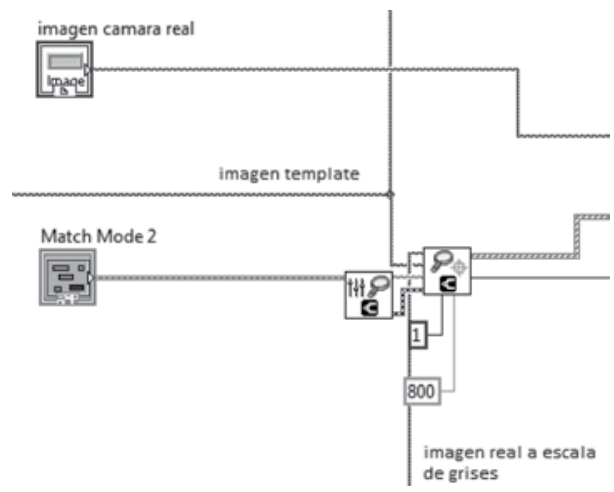

Fig. 28. Proceso para el reconocimiento de señas con el Match Geometric Pattern Fuente: Diego Andrade (autoría)

La imagen real que ingresó en este Sub VI sirve para graficar un recuadro que saldrá en el video en tiempo real cuando la seña sea reconocida; de la misma manera aparecerán avisos y ayudas para facilidad del usuario (véase figura 29).

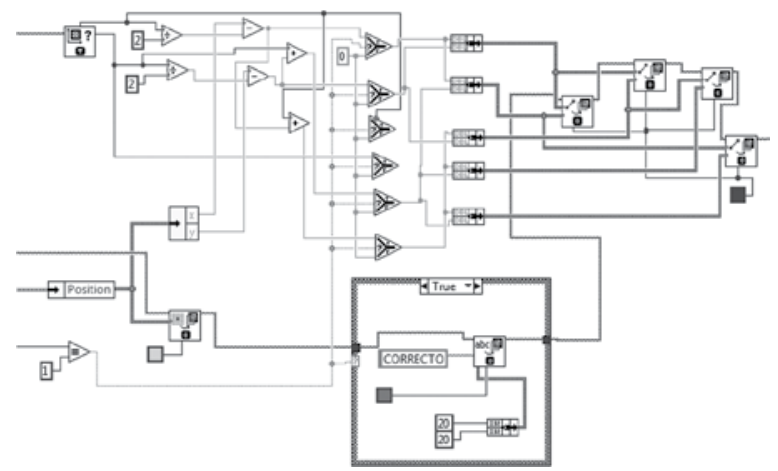

Fig. 29. Proceso de recuadro de señalamiento de la imagen reconocida Fuente: Diego Andrade (autoría)

Dentro del programa principal existe una parte en donde se escribe en un cuadro de texto la letra a la cual corresponde la seña para que la persona sepa, cuando aparezca el recuadro, que la letra fue reconocida (véase figura 30).

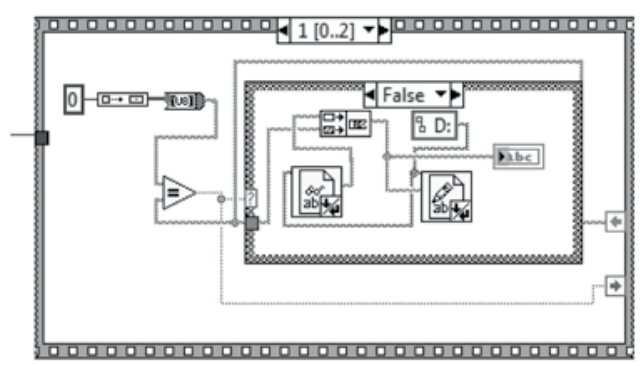

Fig. 30. Proceso de visualización de letra Fuente: Diego Andrade (autoría)

Básicamente la interfaz entre el hombre (discapacidad auditiva) y la máquina (mano robótica), se la implementa en LabView. La persona escoge en el panel del programa una letra del alfabeto, inmediatamente a través de una conexión USB este transmite a nuestro controlador los grados para que sean interpretados y llevados desde el controlador a los servomotores para el movimiento de la mano robótica (véase figura 31).

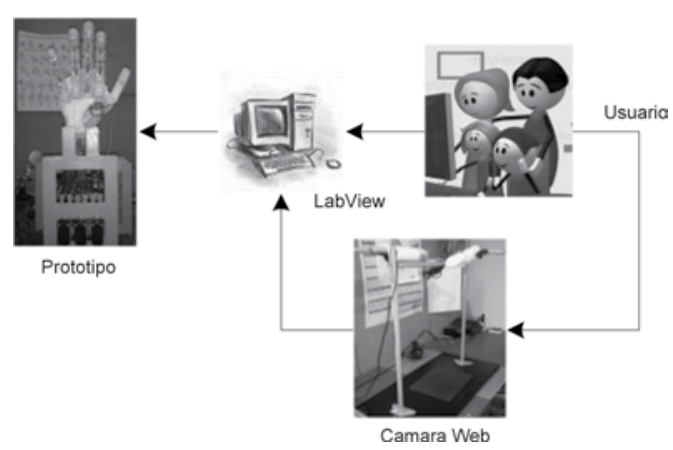

Fig. 31. Interfaz software-hardware Fuente: Diego Andrade (autoría)

Después de realizar la seña, la mano robótica se coloca en posición de reposo. Mientras el programa le pide a la persona que realice la seña que hizo la mano, mediante la cámara web se captan las imágenes y comprueba con los patrones si la seña es la correcta. Existiendo una interacción entre la robótica y el ser humano (véase figura 32). 
La bienvenida del programa interfaz se muestra a continuación:

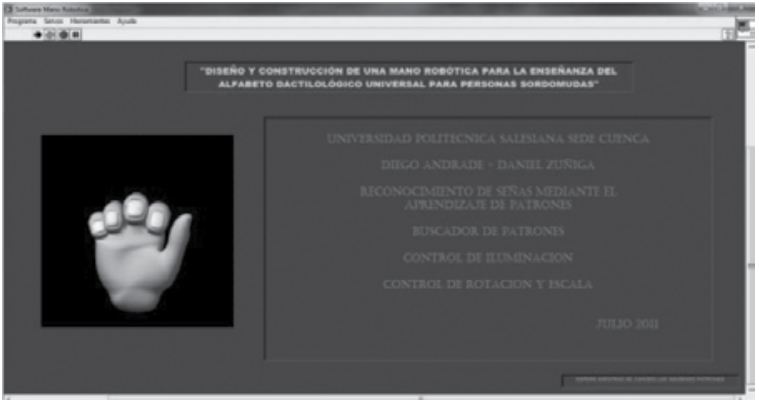

Fig. 32. Inicio del programa interfaz Fuente: Diego Andrade (autoría)

En el panel principal que se muestra en la figura están las letras del alfabeto, la zona de captación de video y las letras o palabras formadas previo reconocimiento de la seña (véase figura 33).

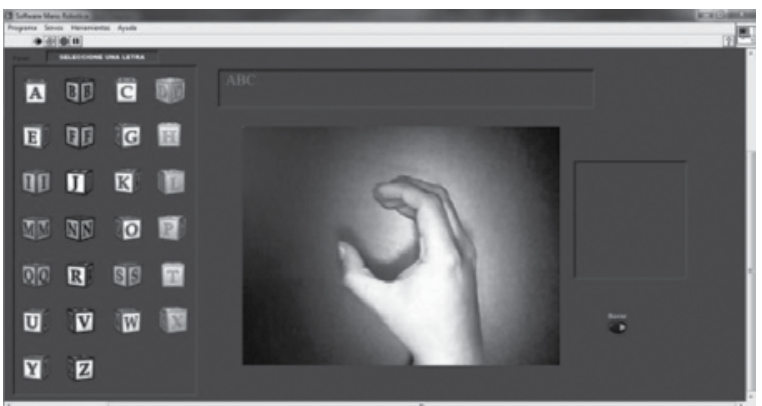

Fig. 33 Programa principal de reconocimiento Fuente: Diego Andrade (autoría)

\section{Resultados}

$\mathrm{Al}$ azar se escogieron tres letras para medir el tiempo que se demora el reconocimiento y el tiempo de aprendizaje (figuras 34, 35, 36). A continuación se muestran las imágenes patrones:

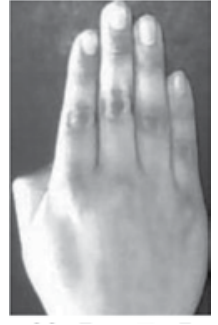

Fig. 34. Patrón letra B

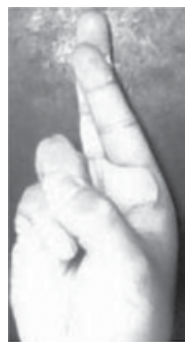

Fuente: Diego Andrade (autoría) Fig. 35. Patrón letra $R$

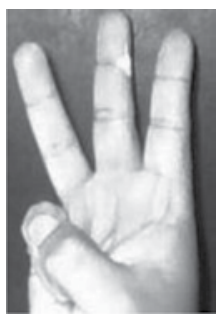

Fuente: Diego Andrade (autoría)

Fig. 36. Patrón letra W

Fuente: Diego Andrade (autoría)

Observando los resultados se muestran en la tabla:

Tabla 3. Estudio antropométrico de la mano por Garret

\begin{tabular}{|c|c|c|c|c|}
\hline Letra & Tiempo1 & Tiempo2 & Tiempo3 & $\begin{array}{c}\text { Tiempo } \\
\text { promedio } \\
\text { Aprendizaje }\end{array}$ \\
\hline $\mathrm{B}$ & $4 \mathrm{~s}$ & $2 \mathrm{~s}$ & $2 \mathrm{~s}$ & $2.66 \mathrm{~s}$ \\
\hline $\mathrm{R}$ & $8 \mathrm{~s}$ & $6 \mathrm{~s}$ & $2 \mathrm{~s}$ & $5.33 \mathrm{~s}$ \\
\hline $\mathrm{W}$ & $6 \mathrm{~s}$ & $5 \mathrm{~s}$ & $3 \mathrm{~s}$ & $4.66 \mathrm{~s}$ \\
\hline
\end{tabular}




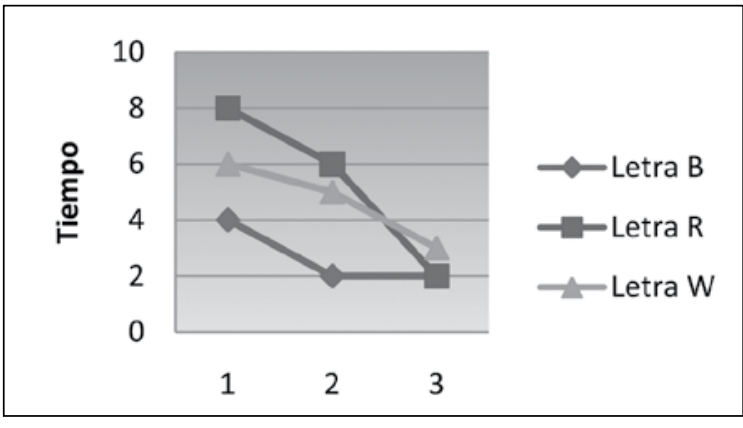

Fig. 37. Tiempo de reconocimiento de señas Fuente: Diego Andrade (autoría)

Examinando los resultados con las pruebas realizadas, se puede ver que conforme la persona interactúe con la mano robótica y aprenda mediante la interfaz, el tiempo que transcurre con respecto a la anterior letra reconocida es menor. Esto da la pauta que el niño o la persona que realice la seña en una forma repetitiva aprende más rápido que viendo en láminas o imágenes. También se puede observar que en la letra $R$ es la que requiere mayor tiempo de aprendizaje por la dificultad que ella tiene (véase figura 37).

\section{Conclusiones}

- El trabajo abarca una sinopsis general del desarrollo realizado sobre el prototipo propuesto y como se evidencia, este robot ha sido desarrollado y será enfocado a ámbitos sociales y educativos.
- Antes de construir cualquier prototipo es importante analizar todas las variables con el objeto de no desperdiciar recursos, en muchos casos costosos.

- El controlar un número alto de variables es complicado, por lo que es recomendado desarrollar algoritmos computaciones para el desarrollo del mismo.

- Como se menciona, los materiales usados para la construcción de este prototipo tienen una gran ventaja en lo que se refiere al costo-beneficio, ya que presentan excelentes características mecánicas y eléctricas.

- Las ecuaciones cinemáticas obtenidas del robot en cuestión proporcionan las variables de control necesarias para adaptar nuestro modelo a cualquier posición en el espacio.

- Al analizar las diferentes variables y los actuadores elegidos nos podemos percatar que es necesario un análisis de fuerzas y de la forma de convertir la energía eléctrica en movimiento.

- Se espera que se promueva la suficiente iniciativa en el país para desarrollar productos de este tipo, que tienen como fin brindar ayuda y asistencia a personas con capacidades especiales. 


\section{Referencias bibliográficas}

[1] Cronología de los robots. En línea: http://sites. google.com/site/anonymousspynet/cronologia

[2] Diario crítico de la comunitat valenciana. En línea: http://www.diariocriticocv.com/noticias/ not133599.htm\#

[3] Warwick K. El cyborg británico. Mundinteractivos, S. A. En línea: http://www.elmundo.es/navegante/2004/11/18/entrevistas/1100776909.html

[4] Wired Science. Mano robótica con sentido pre-táctil. En línea: http://www.tuexperto. com/2008/06/30/mano-robotica-con-sentido-pretactil/

[5] [Tendencias Tecnológicas. Desarrollan una mano robótica impulsada por aire comprimido. En línea: http://www.tendencias21.net/Desarrollan-unamano-robotica-impulsada-por-aire-comprimido_ a3247.html

[6] [Hizook. Una mano robótica de alta velocidad con una destreza increíble. En línea: http://www.tuexperto.com/2009/09/14/una-mano-robotica-de-altavelocidad-con-una-destreza-increible/

[7] En línea: www.pucp.edu.pe/congreso/cibim8/ $\mathrm{pdf} / 25 / 25-13 . \mathrm{pdf}$

[8] En línea: bieec.epn.edu.ec:8180/.../T\%2011213\%20 CAPITULO \%202.pdf

[9] En línea: robots-argentina.com.ar/Actuadores_manos.htm

[10] En línea: anteproyecto-protesis-mano-robotica.googlecode.com/.../25.\%20CWRA_Vivas.pdf
[11] En línea: redalyc.uaemex.mx/redalyc/ pdf/614/61411404.pdf

[12] Semielaborados. En línea: http://www.syar.com. uy/pdf/nth/nth.pdf

[13] Industrias JQ Plásticos de Ingeniería. http://www. jq.com.ar/Imagenes/Productos/Poliamida6/Poliam6/dtecnicos.htm

[14] Materiales aislantes. Nailon - Grilon - Poliamida 6. En línea: http://www.aislantessh.com.ar/aislantes/16.htm

[15] Sánchez O. Cinemática inversa de los manipuladores. Modelos, Control y Sistemas de Visión. En línea: http://omarsanchez.net/Documents/Cinem \%C3\%A1ticainversadelosmanipuladores.pdf

[16] Melo, F. Cinemática directa e inversa de manipuladores robóticos. En línea: http://gaips.inesc-id. pt/ fmelo/Works/R.pdf

[17] Mason, M., Salisbury Jr J. 1985. Robot Hands and the Mechanics of Manipulation. The MIT Press Series in Artificial Intelligence.

[18] Jacobsen, S., Wood, J., Knutti, D., Biggers K. 1986. The UTAH/MIT dextrous hand: Work in progress. In Robot, Grippers, Springer-Verlag, Berlin.

[19] Nakano, Y., Fujie, M., Hosada, Y. 1984. Hitachi's robot hand. Robotics Age, 6(7), julio.

[20] Butterfass, J., Hirzinger, G., Knoch, S., Liu, H. 1998. DLR's multisensory articulated hand. In Proc. of the 1998 IEEE Int. Conf. on Robotics and Automation, Leuven, Bélgica, mayo.

[21] Laschi, C., Dario, P. Grasping and Manipulation in Humanoid Robotics. Scuola Superiore Sant Anna, Italia. 
[22] Lovchik, C., Diftler, M. 1999. The robonaut hand: A dextrous robot hand for space. In Proc. of the 1999 IEEE Int. Conf. on Robotics and Automation, Michigan, mayo.

[23] Schulz, S., Pylatiuk, C. A New Ultralight Anthropomorphic Hand. Inst. of Applied Computer Science Research Center of Karlsruhe, Alemania.

[24] Taylor, G. L., Schwartz R. J. 1955. The Anatomy and Mechanics of the Human Hand, Artificial Limbs, Vol. 2, pp. 22-35.

[25] An, K. N., Chao, E. Y., Cooney, W. P., Linscheid, R. L., 1979. Normative Model of human hand for biomechanical Analisys. Journal of biomechanics, Vol 12. pp. 775-788.

[26] Da Cunha, F., Dynnikov, V. Detalhes construtivos de uma protese antropomorfica para membros superiores - um estudo das transmisões e acionadores. XV congreso brasileiro de engenharia mecanica, 1999 Sao Paulo. Disponible en http://www.sel.eesc.sc.usp.br/labciber/fcunha/ COBEM99.pdf

[27] Dario, P., Lashi, C., Carroza, M. A Human-like Robotic manipulation System Implementing Human Models of Sensory-Motor Coordination, disponible en: http://www-arts.sssup.it/ download/papers/

[28] Ocampo G., Rondón, J. 2004. Diseño y Construcción de una mano robot de cuatro dedos que imite los modelos prensiles humanos. Universidad Militar Nueva Granada.

[29] National Instruments Corporation 